\title{
Hepatitis B Virus Demethylates PD-1 and Modulates the Changes of the Tumor Microenvironment in Hepatocellular Carcinoma Patients
}

\section{Weiguang Lian}

Hebei Medical University

\section{Ruixue Lai}

The Fourth Hospital of Hebei Medical University

Jianhua Wu

The Fourth Hospital of Hebei Medical University

Jingjing Zhang

The Fourth Hospital of Hebei Medical University

\section{Shengchao Li}

The Fourth Hospital of Hebei Medical University

\section{Li Peng}

The Fourth Hospital of Hebei Medical University

\section{Zhanjun Guo ( $\nabla$ zjguo5886@aliyun.com )}

The Fourth Hospital of Hebei Medical University

\section{Research Article}

Keywords: PD-1, DNA methylation, Tumor associated macrophages, Tumor microenvironment, HBV, HCC

Posted Date: August 13th, 2021

DOl: https://doi.org/10.21203/rs.3.rs-793418/v1

License: (9) This work is licensed under a Creative Commons Attribution 4.0 International License. Read Full License 


\section{Abstract}

Background: Hepatitis B virus (HBV) constitutes a major global health burden. Previously study has found the expression of programmed cell death 1 (PD-1) is up-regulated during the chronic HBV infection. However, the mechanism of how HBV infection modulates the expression of PD-1 on CD8 ${ }^{+} \mathrm{T}$ cells are not well understood. In the present study, we aimed to analyzed the role of DNA methylation in regulating the expression of PD-1 on CD $8^{+} \mathrm{T}$ cells during HBV infection, we also aimed to evaluate the HBV induced changes of tumor microenvironment (TME).

Methods: The methylation microarray was used to assess the profile changes of gene methylation upon chronic HBV infection. CD8 ${ }^{+} T$ cells were separated from peripheral blood of health volunteers and HCC patients including HBV related HCC (HBV-HCC) and non-viral HCC. The immune microenvironments of hepatocellular carcinoma (HCC) was interrogated by using Immunofluorescence staining. The PD-1 expression of $\mathrm{CD}^{+} \mathrm{T}$ cells from peripheral blood were examined by western blot and flow cytometry. $\mathrm{T}$ cells function was determined by cytokine measurement. Sequenom MassARRAY platform was used to detected the DNA methylation status of PD-1 promoter.

Results: HBV could drive 413 genes methylated while 3,023 genes including PD-1 demethylated, the reduced PD-1 expression and increased PD-1 demethylation was proved in HBV-HCC tissue. The subsequent analysis indicate that the expression of PD-1 differed by stages of HBV infection, it reduced on the cell membrane of HBV-transfected $\mathrm{CD} 8^{+} \mathrm{T}$ cells upon transient HBV plasmid transfection which might mimic acute HBV infection, while upregulated on the HBV specific $C D 8^{+} T$ cells by demethylation during chronic HBV infection. Interferon-gamma increased in the medium of HBV transfected CD8 ${ }^{+} \mathrm{T}$ cells and interleukin-10 increased in the blood of chronic HBV infection patients. Increased tumor associated macrophage (TAM) cells and T regulatory cells (Tregs) invasion was identified in HBV-HCC tissue when compared with those from non-viral HCC tissue

Conclusion: Our data suggested that the expression of PD-1 varies upon the stage of HBV infection, chronic HBV infection could drove the TME more immunosuppressive through PD-1 demethylation related $\mathrm{CD} 8^{+} \mathrm{T}$ cells exhaustion as well as promotion of invaded Treg and TAM cells thereby to attenuating therapy of immune checkpoint inhibitors.

\section{Background}

Hepatocellular carcinoma (HCC) is the sixth most common cancer worldwide and is responsible for approximately 700,000 deaths annually, making it the fourth leading cause of cancer-related deaths ${ }^{[1]}$. Hepatitis B virus (HBV) or hepatitis C virus infection, alcohol abuse and aflatoxin exposure are the major risk factors for HCC. Of these, HBV infection is the most prominent risk factor for HCC, with over $54 \%$ of HCC patients attributed to HBV ${ }^{[2]}$. Despite the tremendous progress in clinical diagnosis and treatment, the prognosis of $\mathrm{HCC}$ patients remains poor due to the insidious onset and high recurrence rate. Furthermore, the immunosuppressive microenvironment of HCC shows high resistance to currently 
available chemotherapy or molecular targeted drugs ${ }^{[3]}$. Immune checkpoint inhibitors (ICls) that block the programmed death-1 (PD-1) pathway have revolutionized the treatment strategy for advanced HCC. Especially, the combination of atezolizumab plus bevacizumab showed promising efficacy (objective response rate (ORR) $27.3 \%$ vs. $11.9 \%$ ) and prolonged lifespan (median overall survival (OS) 24 vs.11.4 months) than sorafenib alone in first-line treatment for unresectable HCC ${ }^{[4]}$. However, treatment outcomes of anti-PD-1 blockade vary among patients and the disease control rates (DCR) in HBV related $\mathrm{HCC}(\mathrm{HBV}-\mathrm{HCC})$ patients were obviously lower compared with non-viral HCC patients, which imply that HBV infection may compromise the clinical outcomes of HCC patients receiving anti-PD- 1 blockade ${ }^{[5]}$. Thus, characterizing the tumor microenvironment (TME) and epigenetics changes of different etiologies of HCC, particularly those arising from chronic HBV infection, possibly contribute to understand the disease progression.

Hepatitis B virus (HBV), a small hepatotropic DNA virus, chronically infects approximately 260 million people worldwide and constituting a major global health burden ${ }^{[6]}$. HBV is highly prevalent in China, accounting for approximately 93 million HBV carriers and 30 million chronic hepatitis B (CHB) patients ${ }^{[7]}$. Uncontrolled chronic HBV infection could progress to life-threatening terminal-stage chronic liver diseases, such as cirrhosis and HCC ${ }^{[8]}$. DNA methylation, the addition of a group of three hydrogens and a carbon atom to $\mathrm{CpG}$ dinucleotides, could inhibit the access of the transcriptional machinery and cause gene silencing ${ }^{[9]}$. The global demethylation and specific promoter hypermethylation have been linked with genomic instability and inactivation of tumor suppressor genes (TSG), which are drawn into the initiation and proliferation of cancers ${ }^{[10]}$. Studies have suggested that chronic HBV infection could induce and accumulate the aberrant DNA methylation in the pathogenesis of HCC by modulating host DNA methyltransferases and activating a natural kill cell-dependent innate immune response ${ }^{[11-13]}$.

Programmed cell death 1 (PD-1), a receptor of the CD28 superfamily, interacts with its two ligands PD-L1 or PD-L2 and delivers inhibitory signals to T-cells ${ }^{[14]}$. The expression of PD-1 on resting lymphocytes remains at a low basal level to maintain immune tolerance, but transiently up-regulated following an initial immune stimulus ${ }^{[15]}$. During acute antigen exposure, PD-1 expression is down-regulated to stimulate T-cells response for antigen clearance. However, PD-1 expression is maintained at a high level during chronic immune stimulation, leading to T-cells exhaustion ${ }^{[16,17]}$.The exhaustion of T-cells facilitates the escape of virus or tumor cell from the immune surveillance, resulting in the tumorigenesis and viral persistence ${ }^{[18]}$. Blocking the PD-1 or PD-L1 pathway can partially restore the function of T lymphocytes thereby anti-PD1/PD-L1 therapy have shown high response rate and survival benefit in several kinds of cancers ${ }^{[19]}$. HBV-specific $C D 8^{+} T$ cell response is severely impaired in chronic hepatitis $B$ (CHB) patients, leading to long-term immune tolerance and viral persistence ${ }^{[20]}$. In addition, the expression of PD-1 varied depend on the stage of HBV infection, the PD- 1 on HBV-specific CD8 ${ }^{+} \mathrm{T}$ cells is dynamically decreased in acute self-limited hepatitis B patients while up-regulated in CHB patients ${ }^{[21,22]}$. Furthermore, the HBV load is positively correlated with the expression of PD-1 on T cells ${ }^{[22,23]}{ }^{\text {. The TME }}$ in HBV-HCC displayed more immunosuppression than that of non-viral HCC characterized with increased 
PD-1 expressed on the CD8 ${ }^{+} \mathrm{T}$ cells as well as increased T regulatory cells (Tregs) infiltration ${ }^{\text {[22] }}$ However, the mechanism of how HBV infection modulates the expression of PD- 1 on $\mathrm{CD}^{+}{ }^{+} \mathrm{T}$ cells are not well understood.

We utilized Illumina Infinium Human Methylation 450K BeadChip to analyze HBV induced profiles changes of DNA methylation, we found the prompter region of PD-1 in HCC with high HBV load was significantly demethylated (Fig. 1A). We perform our study to illuminate the mechanism of immunocyte exhaustion resulting from HBV induced demethylation of PD-1 as well as the mechanism of HBV induced TME changes.

\section{Materials And Methods}

\section{Patients and Samples}

Tumor tissues and blood samples were collected from HCC patients including HBV- HCC and non-viral HCC who underwent surgical resection at the Fourth Hospital of Hebei Medical University between January 2016 and June 2021. The genomic DNA of tissues and serum were extracted by Wizard Genomic DNA extraction kit (Promega, Madison, WI, USA) according to the manufacturer's protocols. The HBV DNA concentration in HCC tissue was quantified as copies per microgram ( $\mathrm{mg}$ ) of genomic DNA with ABI 7300 TaqMan platform (Life Technologies, Carlsbad, CA, USA) by real-time PCR ${ }^{[24]}$. And the HBV DNA titers in serum was quantified as international units (IU) per milliliter $(\mathrm{ml})$ of genomic DNA with ABI 7500 Fast Real-Time PCR System (Life Technologies, Carlsbad, CA, USA) by real-time PCR. Patients with the HBV DNA load in tissues greater or equal to $6 \times 10^{8} \mathrm{copies} / \mathrm{mg}$ were divided into High-copy HBV group [25], and the HBV DNA load in serum greater or equal to $1 \times 10^{5} \mathrm{IU} / \mathrm{ml}$ were divided into High-copy HBV group. The tumor tissues and blood samples from non-viral HCC were collected as control group. The blood samples of health volunteers were collected for the Lentiviral transduction assay. None of the patients had received antiviral therapy, chemotherapy or molecular targeted drugs prior to surgery. All procedures were supervised and approved by the hospital's ethics committee. Written informed consent was obtained from the participants.

\section{Illumina Infinium Human Methylation 450K BeadChip analysis and methylation assay}

Epigenome-wide DNA methylation was analyzed by using the Illumina Infinium Human Methylation 450K BeadChip array (IIlumina, Inc., San Diego, CA, USA). Briefly, the HCC tissues from High-copy HBV group and control group were collected and the genomic DNA of tissues was extracted by using DNeasy \& Tissue Kit (QIAGEN, Hilden, Germany). A total of 1500 ng DNA was used for bisulfite conversion according to the manufacturer's instructions of the EZ DNA Methylation-Gold Kit (Zymo research, Orange, CA, USA). Bisulfite-converted DNA was analyzed by using Infinium Human Methylation 450K BeadChips 
(IIlumina, Inc., San Diego, CA, USA) according to the manufacturer's protocol ${ }^{[26]}$. BeadChips were scanned using the Illumina Hi-Scan system. Methylation data was processed with Methylation Module (version 1.9.0) of Genome Studio software (Illumina; version 2011.1). DNA methylation measurements with $\mathrm{P}<0.05$ were considered statistically significant.

\section{Cell culture}

Peripheral blood mononuclear cells (PBMCs) were isolated by using Ficoll-Paque (Sigma Chemical Co.St.Louis, MO, USA) density gradient centrifugation from peripheral blood of High-copy HBV group, control group and the healthy volunteers. $\mathrm{CD} 8^{+} \mathrm{T}$ cells were subsequently separated by CliniMACS CD8 (Miltenyi Biotec, Germany) according to the instruction. Then $\mathrm{CD} 8^{+} \mathrm{T}$ cells were activated by Phytohemagglutinin (5ug/ml) (Meilunbio, Dalian, China) and cultured in RPMI-1640 medium (GibcoTM Life Technologies, Grand Island, NY, USA) supplemented with IL-2 (200 IU/mL) (Meilunbio, Dalian, China), 1\% Sodium Pyruvate (GibcoTM Life Technologies, Grand Island, NY, USA), 1\% Glutamine (GibcoTM Life Technologies, Grand Island, NY, USA) and 10\% human $\mathrm{AB}^{+}$serum (Jacques Boy; StemcellTechnologies, Vancouver, $\mathrm{CA}$ ) in a humidified incubator containing $5 \% \mathrm{CO}_{2}$ at $37^{\circ} \mathrm{C}$.

\section{Generation of the lentiviral and construction of HBV infection in T cells}

The plasmids pCS-CG and pCS-HBV1.3 which containing a 1.3-fold-overlength genome of HBV were kindly provided by Professor Xia Chuai from the Department of Microbiology, Hebei Medical University [27]. After packaging with lentivirus package systems, PCS-CG lentivirus and pCS-HBV1.3 lentivirus were transduced into $\mathrm{CD} 8^{+} \mathrm{T}$ cells from health volunteers. The HBV serological markers including hepatitis $\mathrm{B}$ surface antigen (HBsAg) and hepatitis B e antigen (HBeAg) in the culture medium at 48,72 and $120 \mathrm{~h}$ were tested to confirm the successful transfection by the chemiluminescence method on the Cobas e 601analyzer (Roche Diagnostics $\mathrm{GmbH}$, Mannheim, Germany) according to the instruction.

\section{Detection the expression of PD-1 on CD8 ${ }^{+} \mathrm{T}$ cells by Flow cytometric analysis}

The expression of PD-1 on $\mathrm{CD}^{+} \mathrm{T}$ cells was detected by Flow cytometric analysis. $\mathrm{CD} 8^{+} \mathrm{T}$ cells were harvested and washed twice with phosphate buffer (PBS) containing $2 \%$ fetal bovine serum (FBS) (GibcoTM Life Technologies, Grand Island, NY, USA). Then $\mathrm{CD} 8^{+} \mathrm{T}$ cells were incubated with a cocktail of antibodies CD8a-Pacific Blue (Biolegend, San Diego, CA) and PD1-FITC (Biolegend, San Diego, CA) at $4^{\circ} \mathrm{C}$ for $10 \mathrm{~min}$ in the dark. The mixture was subsequently washed once and analyzed by flow cytometer MACSQuant Analyzer 10 (Miltenyi Biotec, Bergisch Gladbach, Germany). 


\section{Cytokine measurement}

The concentrations of cytokine including interleukin-1 $\beta$ (IL-1 $\beta$ ), interferon- $\alpha 2$ (IFN- $\alpha 2)$, IFN- $\gamma$, tumor necrosis factor-a (TNF-a), monocyte chemotactic protein-1 (MCP-1), (IL-6), (IL-8), (IL-10), IL-12p70, IL17A, IL-18, IL-23, and IL-33, were detected by using BioLegend LEGENDplex human inflammation panel (Biolegend, San Diego, CA). The serum from high-copy HBV group and control group, the culture medium from pCS-CG lentivirus group and pCS-HBV1.3 lentivirus group were collected. serum or culture medium of $25 \mu \mathrm{L}$ was diluted 2-fold with Assay Buffer and incubated with $25 \mu \mathrm{L}$ of microbeads and detection antibody each at $25^{\circ} \mathrm{C}$ for 2 hours in the dark. The $25 \mu \mathrm{L}$ Streptavidin-phycoerythrin (SA-PE) was added into each medium subsequently, shaking at approximate $500 \mathrm{rpm}$ for 30 minutes at room temperature in the dark. The flow cytometer MACSQuant Analyzer 10 (Miltenyi Biotec, Bergisch Gladbach, Germany) was used to quantify the PE fluorescence signal of the analyte-specific bead region, and BioLegend's LEGENDplexTM Data Analysis Software (Biolegend, San Diego, CA) was used to generate a standard curve and determine the concentration of the specific analyte. The absolute concentrations in the serum were calculated to known standards.

\section{Western blot analysis}

The protein of HCC tissues collected from High-copy HBV group and control group were extracted by Total Protein Extraction Kit (Invent Biotechnologies, USA) according to the manufacturer's instructions. And the cell protein of $\mathrm{CD} 8^{+} \mathrm{T}$ cells collected from High-copy HBV group and control group were extracted by radio immunoprecipitation assay (RIPA) lysis buffer containing 1\% protease inhibitor (Roche, Basel, Switzerland). Protein extract was subjected to $10 \%$ sodium dodecyl sulphate polyacrylamide gel (SDSPAGE) followed by transfer onto a polyvinylidene difluoride (PVDF) membranes (Roche, Basel, CH). After blocking with $5 \%$ skim milk, the membrane was incubated with primary antibody against human PD1/CD279 (PROTEINTECH, Chicago, USA) or $\beta$-actin (Santa Cruz, CA, USA) overnight at $4^{\circ} \mathrm{C}$, followed by incubation with secondary HRP-conjugated anti-rabbit IgG antibody (Thermo Fisher, New York, USA). The relative intensities of protein bands were visualized with an enhanced chemiluminescence reagent (Thermo Fisher, New York, USA) using the FluorChem ${ }^{\circledR}$ HD2 protein imprinting imaging system (Alpha InnoTec, San Leandro, CA).

\section{Immunofluorescence staining and imaging}

The paraffin tissue sections ( $4 \mu \mathrm{m}$ thick) were baked at $60^{\circ} \mathrm{C}$ for 1 hour and dewaxing with xylene. The tissue sections collected from High-copy HBV group and control group were subsequently rehydrated and subjected to microwave antigen retrieval with citrate buffer $(\mathrm{pH}, 6.0)$ for $30 \mathrm{~min}$. After blocking with $2 \%$ normal goat serum (Invitrogen, California, USA), sections were incubated with primary antibodies including Mouse Monoclonal anti-CD8 (PROTEINTECH, Chicago, USA), Rabbit Polyclonal antiPD1/CD279 (PROTEINTECH, Chicago, USA), CD4 Monoclonal Antibody (ImmunoWay, TX, USA), FOXP3 Polyclonal Antibody (ImmunoWay, TX, USA), CD68 Polyclonal Antibody (ImmunoWay, TX, USA) and 
CD163(ABT-CD163) mouse mAb (ImmunoWay, TX, USA) overnight at $4^{\circ} \mathrm{C}$. Subsequent to washing with PBS $(\mathrm{pH}, 7.2)$ three times, the slides were incubated with a cocktail of Goat Anti Mouse IgG (H\&L) - Alexa Fluor 488 (ImmunoWay, TX, USA) and Goat Anti Rabbit IgG (H\&L) - Alexa Fluor 568 (ImmunoWay, TX, USA) for 1 hours in the dark. After washing with PBS three times, the slides then stained with 4,6diamidino-2-phenylin-dole (DAPI) (Solarbio, Beijing, China) and mounted with Prolong Gold mounting reagent (Life Technologies, Alaska, USA). Then the slides were visualized using the Leica DMi8-M microscope (Leica Microsystems GmbH, Wetzlar, Germany).

\section{Quantitative methylation analysis}

The DNA of CD8 ${ }^{+} \mathrm{T}$ cells and HCC tissues were extracted by using DNeasy \& Tissue Kit (QIAGEN, Germany) according to the manufacturer's instructions. Then, the extracted DNA was subjected to bisulfite conversion and purification by EZ DNA Methylation-Gold Kit (Zymo research, Orange, CA, USA). Quantitative methylation analysis of PD-1 promoter was performed by using the Sequenom MassARRAY platform (CapitalBio, Beijing,China), which was composed of matrix-assisted laser desorption/ionization time-of-flight (MALDITOF) mass spectrometry in combined with RNA base-specific cleavage. PCR primers were designed using Methprimer (http://www.urogene.org/methprimer/), which were listed in TableS1. For each reverse primer, an additional T7 promoter tag for in vivo transcription was added, whereas a 10mer tag on the forward primer was used to adjust melting temperature differences. Mass spectra were obtained via MassARRAY Compact MALDI-TOF (Sequenom) and spectra's methylation ratios were generated using the Epityper software version 1.0 (Sequenom).

\section{Statistical Analysis}

We used descriptive statistics (means and standard deviations or median and ranges) to summarize the data. Statistical analyses were performed by $\chi 2$ test or Student's $t$ test. $P<0.05$ was considered statistically significant difference. Data was obtained from at least three independent experiments with a similar pattern. All statistical analyses were carried out using SPSS statistical software, version 19.0 (IBM Corporation, Armonk, NY).

\section{Results}

\section{HBV increases PD-1 expression by demethylating the promoter region of PD-1.}

To investigate the alteration of DNA methylation statues induced by HBV infection, the methylation microarray analysis was performed with genomic DNA extracted from high HBV load and non-viral HCC tissues (Fig. 1A). A total of 14,454 differentially methylated CpG sites (covering 3,436 genes) were detected, with 581 sites (covering 413 genes) were hypermethylated and 13,873 sites (covering 3,023 genes) were demethylated (Fig. 1B) by comparing high HBV load and non-viral HCC. These data suggested that the widely demethylation occurred upon chronic HBV infection. In addition, GO annotation (Fig. 1C) and KEGG (Fig. 2A) pathway enrichment analyses were employed to obtain a deeper insight into the biological roles of these differentially methylated genes. GO Biological Process analysis (Fig. 1C) revealed that these differentially methylated genes were markedly enriched in the nervous system 
development, system development, and single-multicellular organism process. For GO Cellular Component analysis (Fig. 1C), the top three significantly enriched terms were cell periphery, plasma membrane, and plasma membrane part. The top three significantly enriched Molecular Function terms included gated channel activity, ion channel activity and substrate-specific channel activity (Fig. 1C). Furthermore, the top three markedly enriched pathways for these differentially methylated genes were Glutamatergic synapse, Morphine addiction, and Retrograde endocannabinoid signaling (Fig. 2A). Among the changes of gene methylation profiles, the demethylation of PD-1 in HBV-related HCC patients was found by compared with those of non-viral HCC patients. The demethylation of PD-1 prompter region was also confirmed by MassARRAY with genomic DNA from HCC tissue (site 2nd, 8th, 10th, 11th ,12 $12^{\mathrm{ve}}, 13 \mathrm{rd}, \mathrm{P}$ $<0.05$ ) (overall methylation levels, $p<0.01$ ) (Fig. 2B). In addition, the expression level of PD-1 protein in $\mathrm{HBV}$-related HCC is also significantly up-regulated by our measurement (Fig. 2C). All of above implied that chronic HBV infection increased the PD-1 expression by demethylating the promoter region of PD-1.

\section{The influence of HBV induced PD-1 methylation on CD8 + T cell and TME of HCC patients}

Since PD-1 expressed majorly in CD8 ${ }^{+} T$ cells, we evaluated the HBV mediated PD-1 expressional changes in $C D 8^{+} T$ cells. The flow cytometric analysis was used to detect the PD- 1 form surface of $C D 8^{+} T$ cells with blood of HCC patients. The mean fluorescent intensity (MFI) of PD-1 on CD8 ${ }^{+} T$ cells $(73.53 \pm 16.98$ vs. $56.97 \pm 6.57, p=0.004)$ and the percentage of $P D 1^{+} C D 8^{+} T$ cell $(17.55 \pm 13.58 \%$ vs. $5.43 \pm 3.76 \%, p=$ 0.042) in High-copy HBV group were significantly upregulate compared with that of control group (Fig. $3 A)$. In addition, the expression level of PD-1 protein in CD8 ${ }^{+} \mathrm{T}$ cells from blood of HBV-HCC patients is also significantly up-regulated (Fig. 3B). And the promoter PD- 1 from $\mathrm{CD}^{+} \mathrm{T}$ cells of HBV blood shew significant demethylation by MassARRAY analysis (site 1st,3-4th ,5th ,14th, $\mathrm{P}<0.05$ ) (overall methylation levels, $p<0.05$ ) (Fig. 3C). Thus, the aforementioned findings indicate that chronic HBV infection may upregulate the expression of PD- 1 from $\mathrm{CD}^{+} \mathrm{T}$ cells through demethylating PD-1 promoter. Although the statistical changes referring to cytokines from HBV blood was not reached, we found cytokines IL-10 $(167.73 \pm 66.621 v s .61 .543 \pm 32.233, p=0.067)$ displayed an upward trend at borderline difference level (Fig. 3D and S1).

To illustrates the potential TME alteration caused by HBV infection, the immune landscape for HCC tissues with high HBV load and non-viral HCC tissues were detected by immunofluorescence method. The infiltration of $\mathrm{PD} 1^{+} \mathrm{CD} 8^{+} \mathrm{T}$ cells were detected by using Mouse Monoclonal anti-CD8 antibody and Rabbit Polyclonal anti-PD1/CD279 antibody. Treg differentiation was identified and quantified using immunostaining for CD4 Monoclonal Antibody and FOXP3 Polyclonal Antibody. And CD68 Polyclonal Antibody and CD163(ABT-CD163) mouse mAb were used to quantitative assessment of tumor-associated macrophages (TAM). The expression of PD-1 on the surface of tumor infiltration $\mathrm{CD}^{+}{ }^{+} \mathrm{T}$ cells was significantly upregulated in HBV-HCC tissue (Fig. 4). Furthermore, the percentages of infiltrating Treg and TAM cells in HBV-HCC tissues was statistically higher compared with non-viral HCC tissues (Fig. 4). All of above demonstrated that the HBV could induce inhibitory TME changes with increased TAM and Treg invading in HBV-HCC patients. 


\section{The expressional change of PD-1 on cell membrane of $\mathrm{CD} 8{ }^{+} \mathrm{T}$ cells upon in vitro HBV infection.}

The pCS-CG lentivirus and PCS-HBV1.3 lentivirus were transduced into CD8 ${ }^{+} \mathrm{T}$ cells of healthy volunteers to evaluate the HBV related PD-1 changes. The chemiluminescence method was used to detect the HBV serological markers including HBsAg and HBeAg so as to confirm the successful HBV transfection. HBsAg $(p=0.003)$ and HBeAg $(p<0.001)$ were upregulated in pCS-HBV1.3 groups compared with pCS-CG groups at the timepoint of 48 hours after transfection (Table S2). The PD-1 on the cell membrane of CD $8^{+}$ $T$ cells decreased significantly upon transient HBV transfection by flow cytometric analysis $(p<0.05)$ (Fig. $5 A)$. In addition, the methylation levels of PD-1 promoter weren't changed after 48 hours of HBV plasmid transfection (Fig. 5B). The cytokines in the culture medium of $\mathrm{CD}^{+} \mathrm{T}$ cell was detected after 48 hours of HBV plasmid transfection to identify PD-1 reduction related cytokines, the increased cytokine of IFN- $y$ was found by flow cytometric analysis $(p=0.012)$ (Fig. $5 C$ and S2). These data demonstrated that HBV transient transfection would activate $\mathrm{CD}^{+} \mathrm{T}$ cells through decreasing PD-1 expression without methylation changes as well as increasing IFN-ץ levels.

\section{Discussion}

About $90 \%$ of people exposed to HBV in early childhood develop chronic infection, the continued HBV infection would initiate decades of process for cirrhosis even HCC ${ }^{[28]}$, so almost all HBV-HCC patients are in a state of chronic HBV infection. PD-1 is a key feature of T cell exhaustion with expressing on the over $90 \%$ of liver-infiltrating HBV-specific CD $8^{+} T$ cells ${ }^{[29,30]}$, it dephosphorylated the costimulatory receptor CD28 and other T cell receptor (TCR) associated component to upregulate the inhibitory genes at initiation of $C D 8^{+} T$ cells exhaustion ${ }^{[31,32]}$. The chronic virus infection including HBV infection could increase the expression of PD-1 thereby to initiate the exhaustion of $\mathrm{CD}^{+}{ }^{+}$T cells ${ }^{[30]}$. In the present study, we found that the HBV induced demethylation of PD-1 would be responsible for the PD- 1 initiated $C D 8^{+} T$ cells exhaustion in HBV-HCC patients. We also found that HBV could change the TME in HCC tissue through increasing TAM cells and Treg cells. Our data implied that HBV infection might reduce the efficiency of ICl through promoting the $\mathrm{CD} 8^{+} \mathrm{T}$ cells exhaustion as well as increasing TAM and Treg cells.

HBV infection induced host DNA abnormal methylation to facilitate its evading from host defense and to promote its own persistence in chronic HBV infection ${ }^{[33]}$. Meanwhile, the cancer cell genomes in HBVHCC undergo global demethylation due to HBV X protein $(\mathrm{HBx})$ induced downregulation of DNA methyltransferase 3B (DNMT3B), which contribute to the genomic instability and oncogenes activation $(34,35)$. Our data of methylation microarray supported the presumption that chronic HBV infection could initiate the widely DNA demethylation in genome of HCC cell.

Our data demonstrated that Tregs was more enriched in HBV- HCC compared with non-viral HCC, which was consistent with the previous studies (22). HBV infection also increased Treg activity through demethylating the Treg-specific demethylated region (TSDR) ${ }^{[34]}$.. Macrophages derived from bone marrow-derived circulating monocytes are highly plastic in response to complex stimuli. The activated 
macrophages (M1) can kill and remove tumor cells, while the M2 macrophages, also considered as tumor-associated macrophages (TAMs), are usually educated by tumor cells to promote tumor immune escape, angiogenesis, tumor growth, and metastasis ${ }^{[35]}$. In our study, we found HBV promoted more M2 macrophages in tissue of HBV-HCC, but the true mechanism how HBV inducing TAM differentiation need to be evaluated in the future. HBV induced immunosuppressive TME changes including increased $C D 8^{+} T$ cells exhaustion as well as Tregs and M2 infiltration would make HBV-HCC more resistant for ICls. We also found HBV related IL-10 changes in blood of HBV-HCC patients, previous experiments in vitro with monocyte and natural killer (NK) cells showed that HBV promote IL-10 secretion by upregulating nuclear factor kappa-B (NF-KB) and myeloid differentiation factor 88 (MyD88) ${ }^{[36]}$. IL-10 overexpression might impair the antiviral response of IFN-a by inhibiting the STAT1-Tyr701 phosphorylation on macrophages and result in the viral persistence ${ }^{[37]}$. Moreover, IL-10 increases PD-L1 expression on macrophage colonystimulating factor-derived dendritic cells (M-DC), which in turn induces the expression of PD- 1 on $C D 8^{+} T$

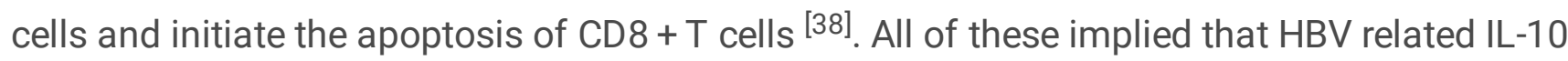
overproduction enhanced immunosuppression of TME in HBV-HCC patients, the potential interaction among $\mathrm{CD} 8^{+} \mathrm{T}$ cells exhaustion, TAM and Treg promotion, and IL-10 overexpression need to be furtherly illustrated.

Transient infection of $\mathrm{HBV}$ into $\mathrm{CD} 8^{+} \mathrm{T}$ cell induced the reduction of PD-1 expression on $\mathrm{CD} 8^{+} \mathrm{T}$ membrane and the increase of cytokine IFN- $\gamma$ secretion, which might mimic the process of acute HBV infection. The reduction of immunosuppressive PD- 1 on $\mathrm{CD} 8^{+} \mathrm{T}$ cell membrane could activate $\mathrm{CD} 8^{+} \mathrm{T}$ cell thereby to favor HBV clearance. And previous study has shown that the PD-1 dynamic decrease was correlated with $\mathrm{HBV}$-specific effector $\mathrm{CD} 8^{+} \mathrm{T}$ cells developing into memory $\mathrm{T}$ cells during the phase of acute HBV infection ${ }^{[39]}$.

\section{Conclusion}

Our data suggested that the expression of PD-1 varies upon the stage of HBV infection, chronic HBV infection could drove the TME more immunosuppressive through PD-1 demethylation related CD8 ${ }^{+} \mathrm{T}$ cells exhaustion as well as promotion of invaded Treg and TAM cells thereby to attenuating therapy of immune checkpoint inhibitors (ICls).

\section{Abbreviations}

HBV: Hepatitis B virus, PD-1: programmed cell death 1, TME:vtumor microenvironment, HBV-HCC: HBV related HCC, HCC: hepatocellular carcinoma, TAM: tumor associated macrophage, Tregs: T regulatory cells, ICls: Immune checkpoint inhibitors, ORR: objective response rate, OS: overall survival, DCR: disease control rates, CHB: chronic hepatitis B, TSG: tumor suppressor genes, mg: microgram, IU: international units, ml: milliliter, PBMCs: Peripheral blood mononuclear cells, HBsAg: hepatitis B surface antigen, HBeAg: hepatitis B e antigen, PBS: phosphate buffer, FBS: fetal bovine serum, IL-1 1 : interleukin-1 $\beta$, IFNa2: interferon- $\alpha 2$, TNF-a: tumor necrosis factor-a, MCP-1: monocyte chemotactic protein-1, SA-PE: 
Streptavidin-phycoerythrin, RIPA; radio immunoprecipitation assay, SDS-PAGE: sodium dodecyl sulphate polyacrylamide gel, PVDF: polyvinylidene difluoride membranes, MFI: mean fluorescent intensity, HBx: HBV X protein, DNMT3B: DNA methyltransferase 3B, TSDR: Treg-specific demethylated region, NK: natural killer cells, NF-kB: nuclear factor kappa-B, MyD88: myeloid differentiation factor 88.

\section{Declarations}

\section{Ethics approval and consent to participate}

All procedures were supervised and approved by the hospital's ethics committee (2018MEC013). Written informed consent was obtained from the participants.

\section{Consent for publication}

Not applicable.

\section{Availability of data and materials}

The original contributions presented in the study are included in the article/Supplementary Material. Further inquiries can be directed to the corresponding authors.

\section{Competing interests}

The authors declare that they have no competing interests.

\section{Funding}

This work was supported by the Natural Science Foundation of China of Hebei Province (Grant No. $\mathrm{H} 2019206428)$.

\section{Authors' Contributions}

WL and RL performed all experiments, JW collected data, JZ and SL performed statistical analysis, ZG and LP designed the experiments, conceived the concept, and wrote the manuscript. All authors had final approval of the submitted and published versions.

\section{Acknowledgments}

We thank Professor Xia Chuai (Department of Microbiology, Hebei Medical University) for the plasmid.

\section{References}

1. Bray F, Ferlay J, Soerjomataram I, et al. Global cancer statistics 2018: GLOBOCAN estimates of incidence and mortality worldwide for 36 cancers in 185 countries. CA Cancer J Clin. 2018;68(6):394424. 
2. EASL Clinical Practice Guidelines: Management of hepatocellular carcinoma. J Hepatol. 2018;69(1):182-236.

3. Jindal A, Thadi A, Shailubhai K. Hepatocellular Carcinoma: Etiology and Current and Future Drugs. J Clin Exp Hepatol. 2019;9(2):221-32.

4. Finn RS, Qin S, Ikeda M, et al. Atezolizumab plus Bevacizumab in Unresectable Hepatocellular Carcinoma. N Engl J Med. 2020;382(20):1894-905.

5. Li B, Yan C, Zhu J, et al. Anti-PD-1/PD-L1 Blockade Immunotherapy Employed in Treating Hepatitis B Virus Infection-Related Advanced Hepatocellular Carcinoma: A Literature Review. Front Immunol. 2020;11:1037.

6. Stanaway JD, Flaxman AD, Naghavi M, et al. The global burden of viral hepatitis from 1990 to 2013: findings from the Global Burden of Disease Study 2013. Lancet. 2016;388(10049):1081-8.

7. Lu FM, Zhuang H. Management of hepatitis B in China. Chin Med J (Engl). 2009;122(1):3-4.

8. Jia $\mathrm{L}, \mathrm{Gao} Y, \mathrm{He} Y$, et al. HBV induced hepatocellular carcinoma and related potential immunotherapy. Pharmacol Res. 2020;159:104992.

9. Black JC, Whetstine JR. Chromatin landscape: methylation beyond transcription. Epigenetics. 2011;6(1):9-15.

10. Pan Y, Liu G, Zhou F, et al. DNA methylation profiles in cancer diagnosis and therapeutics. Clin Exp Med. 2018;18(1):1-14.

11. Kuss-Duerkop SK, Westrich JA, Pyeon D. DNA Tumor Virus Regulation of Host DNA Methylation and Its Implications for Immune Evasion and Oncogenesis. Viruses. 2018;10(2).

12. Okamoto $Y$, Shinjo $K$, Shimizu $Y$, et al. Hepatitis virus infection affects DNA methylation in mice with humanized livers. Gastroenterology. 2014;146(2):562-72.

13. Ye C, Tao R, Cao Q, et al. Whole-genome DNA methylation and hydroxymethylation profiling for HBVrelated hepatocellular carcinoma. Int J Oncol. 2016;49(2):589-602.

14. Sharpe AH, Freeman GJ. The B7-CD28 superfamily. Nat Rev Immunol. 2002;2(2):116-26.

15. Agata $Y$, Kawasaki A, Nishimura $H$, et al. Expression of the PD-1 antigen on the surface of stimulated mouse T and B lymphocytes. Int Immunol. 1996;8(5):765-72.

16. Day CL, Kaufmann DE, Kiepiela P, et al. PD-1 expression on HIV-specific T cells is associated with Tcell exhaustion and disease progression. Nature. 2006;443(7109):350-4.

17. Urbani $S$, Amadei $B$, Tola $D$, et al. PD-1 expression in acute hepatitis $C$ virus (HCV) infection is associated with HCV-specific CD8 exhaustion. J Virol. 2006;80(22):11398-403.

18. Bally AP, Austin JW, Boss JM. Genetic and Epigenetic Regulation of PD-1 Expression. J Immunol. 2016;196(6):2431-7.

19. Lei Q, Wang D, Sun K, et al. Resistance Mechanisms of Anti-PD1/PDL1 Therapy in Solid Tumors. Front Cell Dev Biol. 2020;8:672.

20. Boeijen LL, Hoogeveen RC, Boonstra A, et al. Hepatitis B virus infection and the immune response: The big questions. Best Pract Res Clin Gastroenterol. 2017;31(3):265-72. 
21. Zhang Z, Zhang JY, Wherry EJ, et al. Dynamic programmed death 1 expression by virus-specific CD8 T cells correlates with the outcome of acute hepatitis B. Gastroenterology. 2008;134(7):1938-49, 49.e1-3.

22. Lim CJ, Lee $\mathrm{YH}$, Pan $\mathrm{L}$, et al. Multidimensional analyses reveal distinct immune microenvironment in hepatitis B virus-related hepatocellular carcinoma. Gut. 2019;68(5):916-27.

23. Hou FQ, Wu XJ, Wang Y, et al. Rapid downregulation of programmed death-1 and interferon- $Y$ inducible protein-10 expression is associated with favourable outcome during antiviral treatment of chronic hepatitis B. J Viral Hepat. 2013;20 Suppl 1:18-26.

24. Peng L, Yang G, Wu C, et al. Mutations in hepatitis B virus small S genes predict postoperative survival in hepatocellular carcinoma. Onco Targets Ther. 2016;9:7367-72.

25. Chen J, Yin J, Tan X, et al. Improved multiplex-PCR to identify hepatitis B virus genotypes A-F and subgenotypes B1, B2, C1 and C2. J Clin Virol. 2007;38(3):238-43.

26. Bibikova M, Barnes B, Tsan C, et al. High density DNA methylation array with single CpG site resolution. Genomics. 2011;98(4):288-95.

27. Chuai $X$, Wang $W$, Chen $H$, et al. Lentiviral backbone-based hepatitis $B$ virus replicon-mediated transfer favours the establishment of persistent hepatitis $B$ virus infection in mice after hydrodynamic injection. Antiviral Res. 2014;101:68-74.

28. Wu J, Han M, Li J, et al. Immunopathogenesis of HBV Infection. Adv Exp Med Biol. 2020;1179:71107.

29. Wherry EJ, Kurachi M. Molecular and cellular insights into T cell exhaustion. Nat Rev Immunol. 2015;15(8):486-99.

30. Bertoletti A, Ferrari C. Adaptive immunity in HBV infection. J Hepatol. 2016;64(1 Suppl):S71-s83.

31. Yokosuka T, Takamatsu M, Kobayashi-Imanishi W, et al. Programmed cell death 1 forms negative costimulatory microclusters that directly inhibit $\mathrm{T}$ cell receptor signaling by recruiting phosphatase SHP2. J Exp Med. 2012;209(6):1201-17.

32. Hui E, Cheung J, Zhu J, et al. T cell costimulatory receptor CD28 is a primary target for PD-1mediated inhibition. Science. 2017;355(6332):1428-33.

33. Hensel KO, Rendon JC, Navas MC, et al. Virus-host interplay in hepatitis B virus infection and epigenetic treatment strategies. Febs j. 2017;284(21):3550-72.

34. Liu HR, Li WM. Treg-specific demethylated region activity in isolated regulatory $t$ lymphocytes is a surrogate for disease severity in hepatocellular carcinoma. IUBMB Life. 2015;67(5):355-60.

35. Kim J, Bae JS. Tumor-Associated Macrophages and Neutrophils in Tumor Microenvironment. Mediators Inflamm. 2016;2016:6058147.

36. Li H, Zhai N, Wang Z, et al. Regulatory NK cells mediated between immunosuppressive monocytes and dysfunctional T cells in chronic HBV infection. Gut. 2018;67(11):2035-44.

37. Song H, Tan G, Yang Y, et al. Hepatitis B Virus-Induced Imbalance of Inflammatory and Antiviral Signaling by Differential Phosphorylation of STAT1 in Human Monocytes. J Immunol. 
2019;202(8):2266-75.

38. Kim YJ, Park SJ, Broxmeyer HE. Phagocytosis, a potential mechanism for myeloid-derived suppressor cell regulation of CD8+ T cell function mediated through programmed cell death-1 and programmed cell death-1 ligand interaction. J Immunol. 2011;187(5):2291-301.

39. Zhang Z, Jin B, Zhang JY, et al. Dynamic decrease in PD-1 expression correlates with HBV-specific memory CD8 T-cell development in acute self-limited hepatitis B patients. J Hepatol. 2009;50(6):1163-73.

\section{Figures}

A
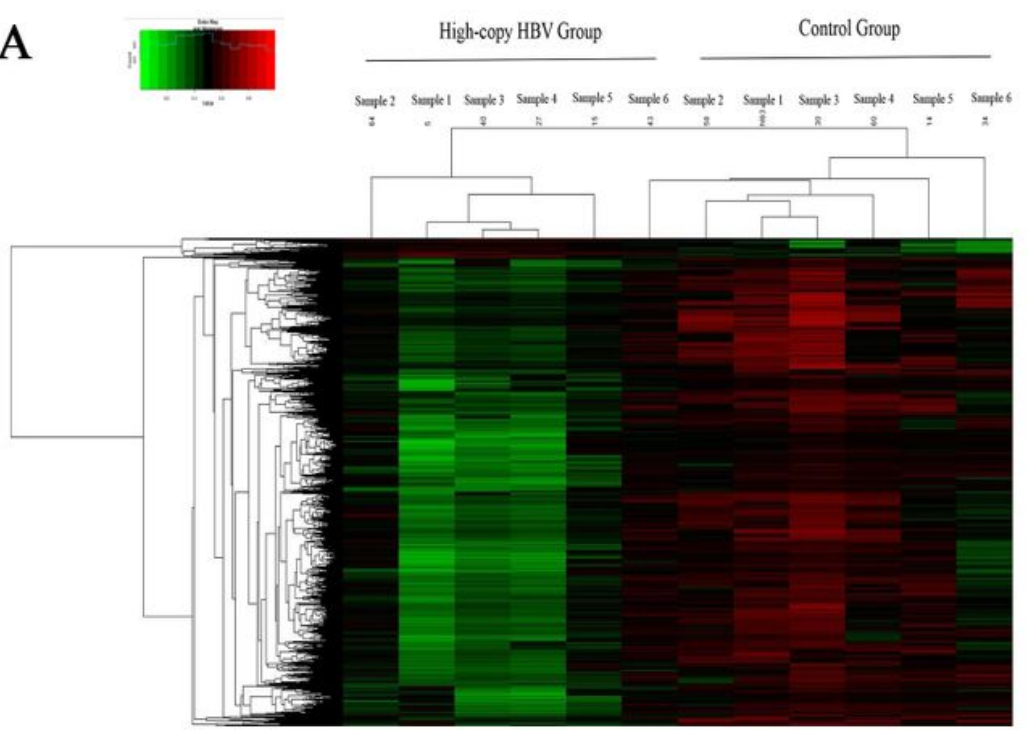

\section{B}

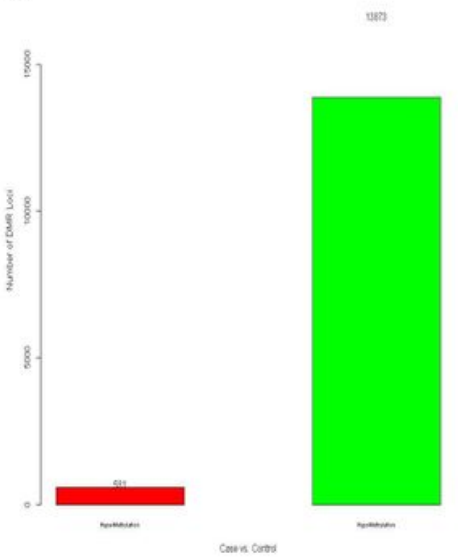

C

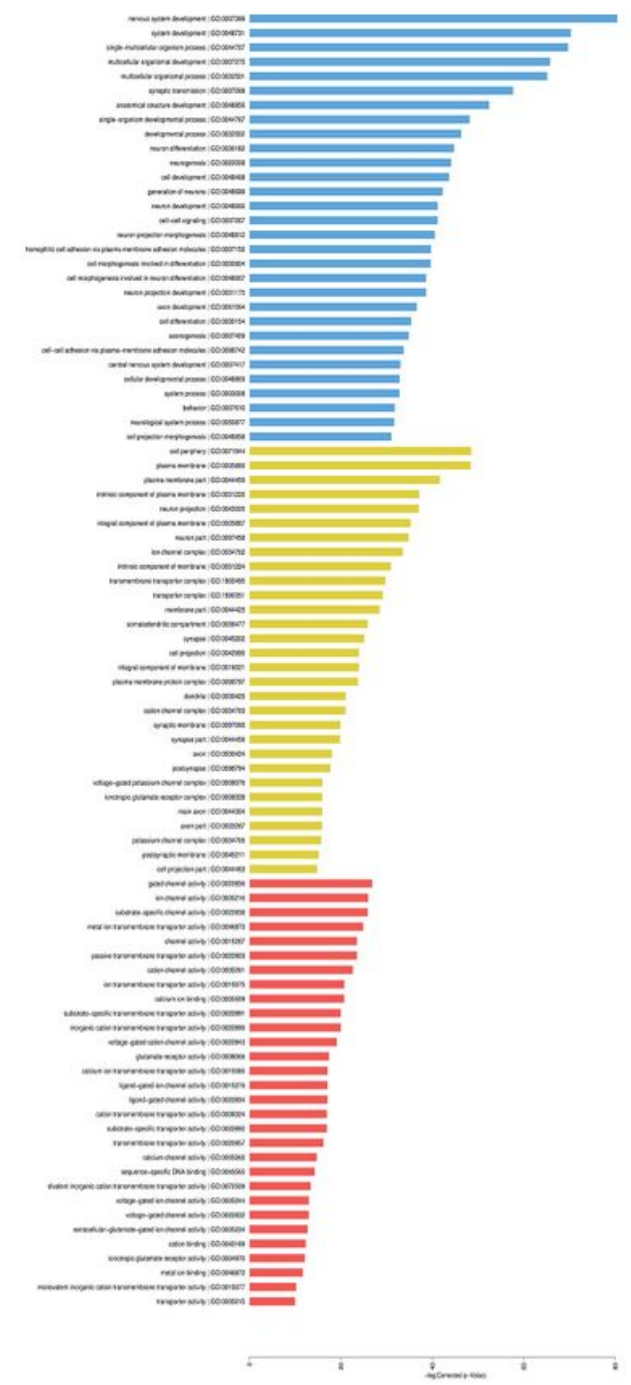

\section{Figure 1}

CpG methylation analysis A. Cluster analysis of different gene methylation between high-copy group and control group; B. Numbers of differentially methylated sites and genes between high-copy group and 
control group; C. GO Barplot.

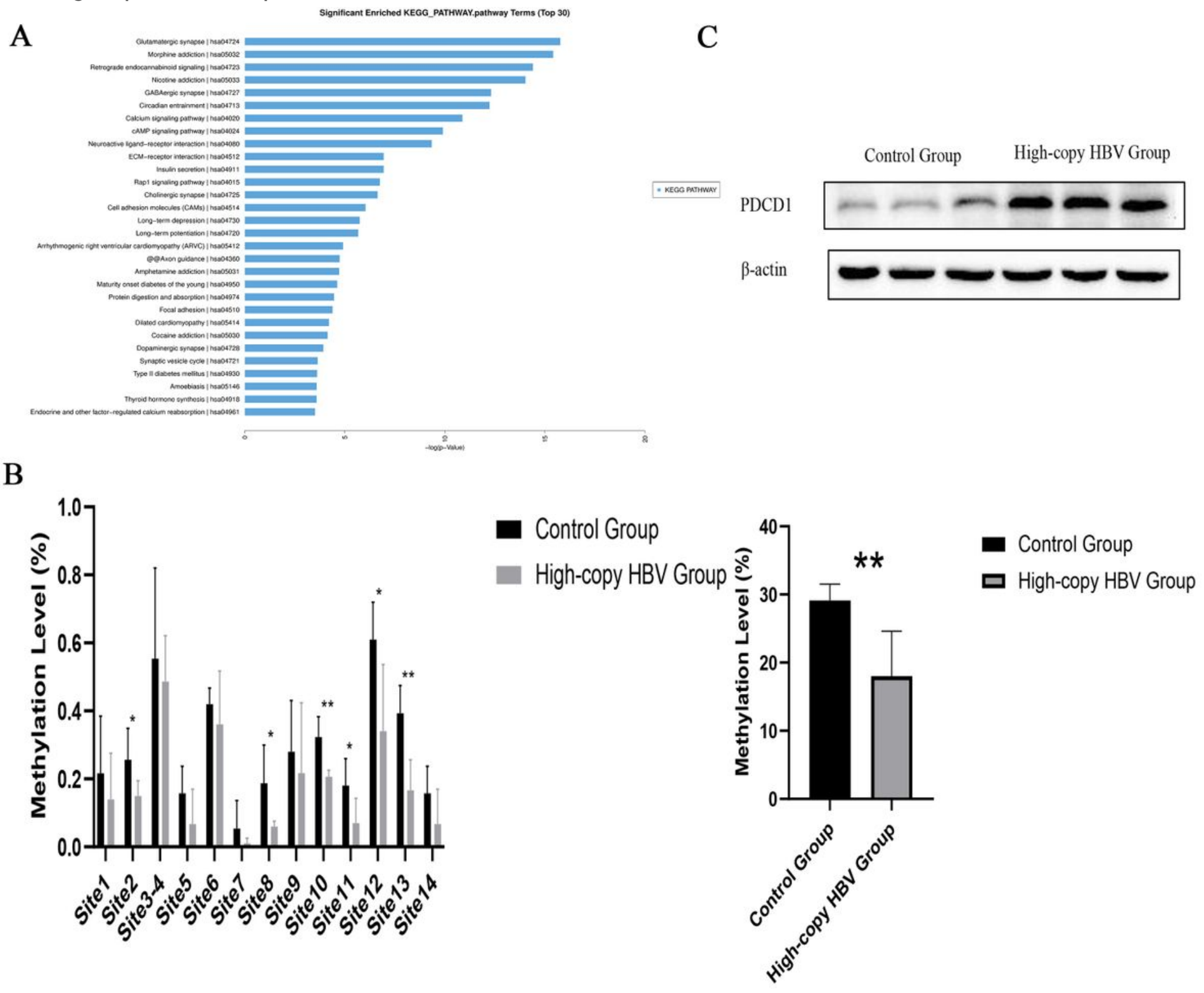

Figure 2

HBV-mediated demethylation of PD-1 DNA promoter leads to enhanced PD-1 expression in HCC tissues A. KEGG Barplot; B. Sequenom MassARRAY analysis of PD-1 gene methylation in high-copy HBV infection group and non-HBV infection group; C. Western blot analysis of PD-1 protein in high-copy HBV infection group and non-HBV infection group; ${ }^{*}<0.05,{ }^{* *}<0.01$. 
A
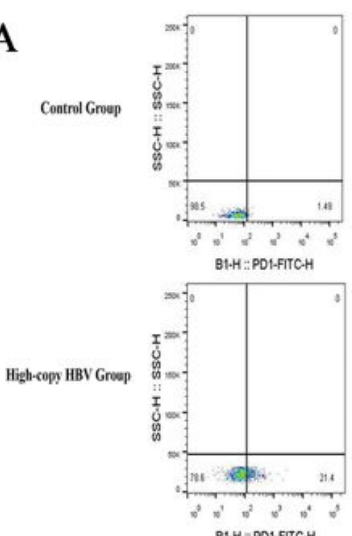

B1.H $=$ PO1.FICH
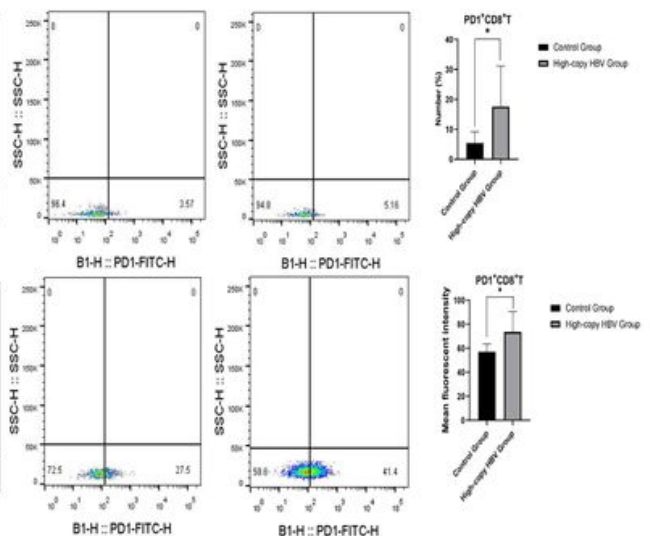

B

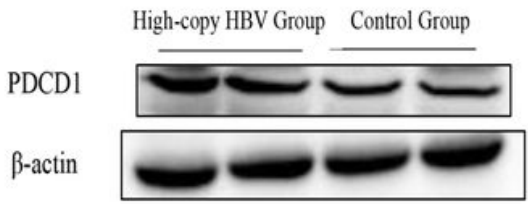

C
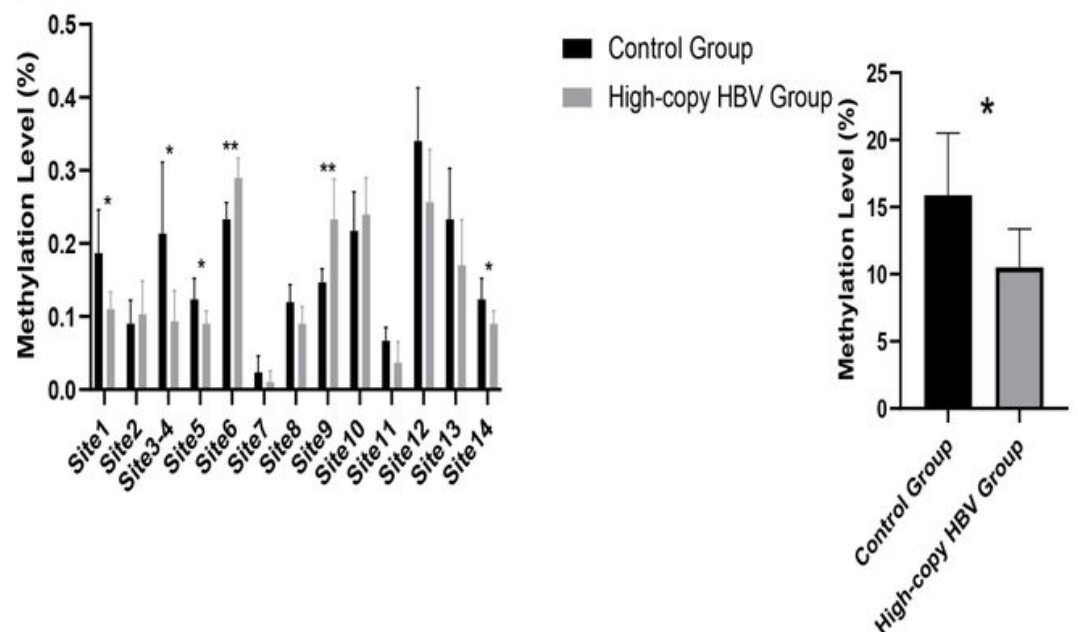

D

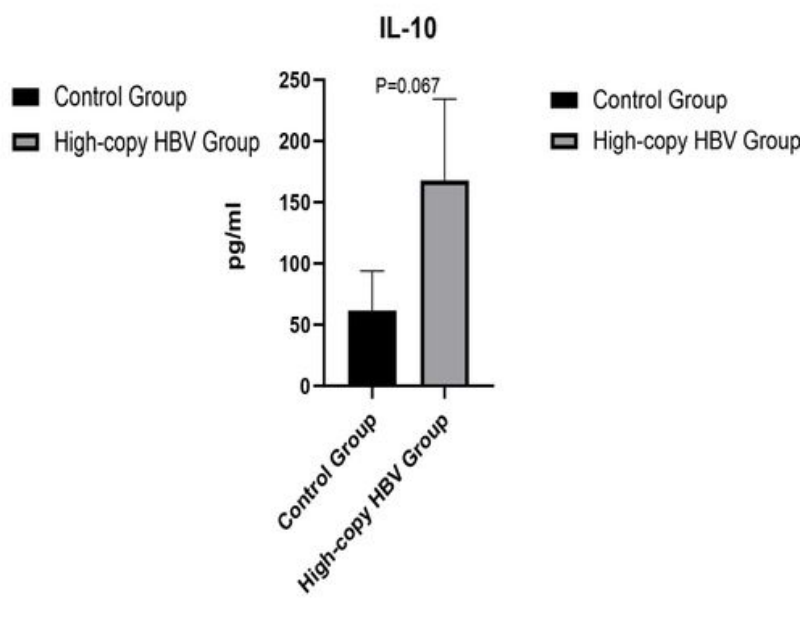

Figure 3

HBV infection up-regulated the expression of PD-1 on CD8+ T cells through DNA demethylation in HCC patients. A. Detection the expression of PD-1 on CD8+ T cells by Flow cytometric analysis in HBV- HCC group and non-viral HCC group; B. Western blot analysis of PD-1 protein in HBV- HCC group and non-viral HCC group; C. Sequenom MassARRAY analysis of PD-1 gene methylation in HBV- HCC group and nonviral HCC group; D. Cytokine measurement in HBV- HCC group and non-viral HCC group; $*<0.05, * \star<0.01$. 

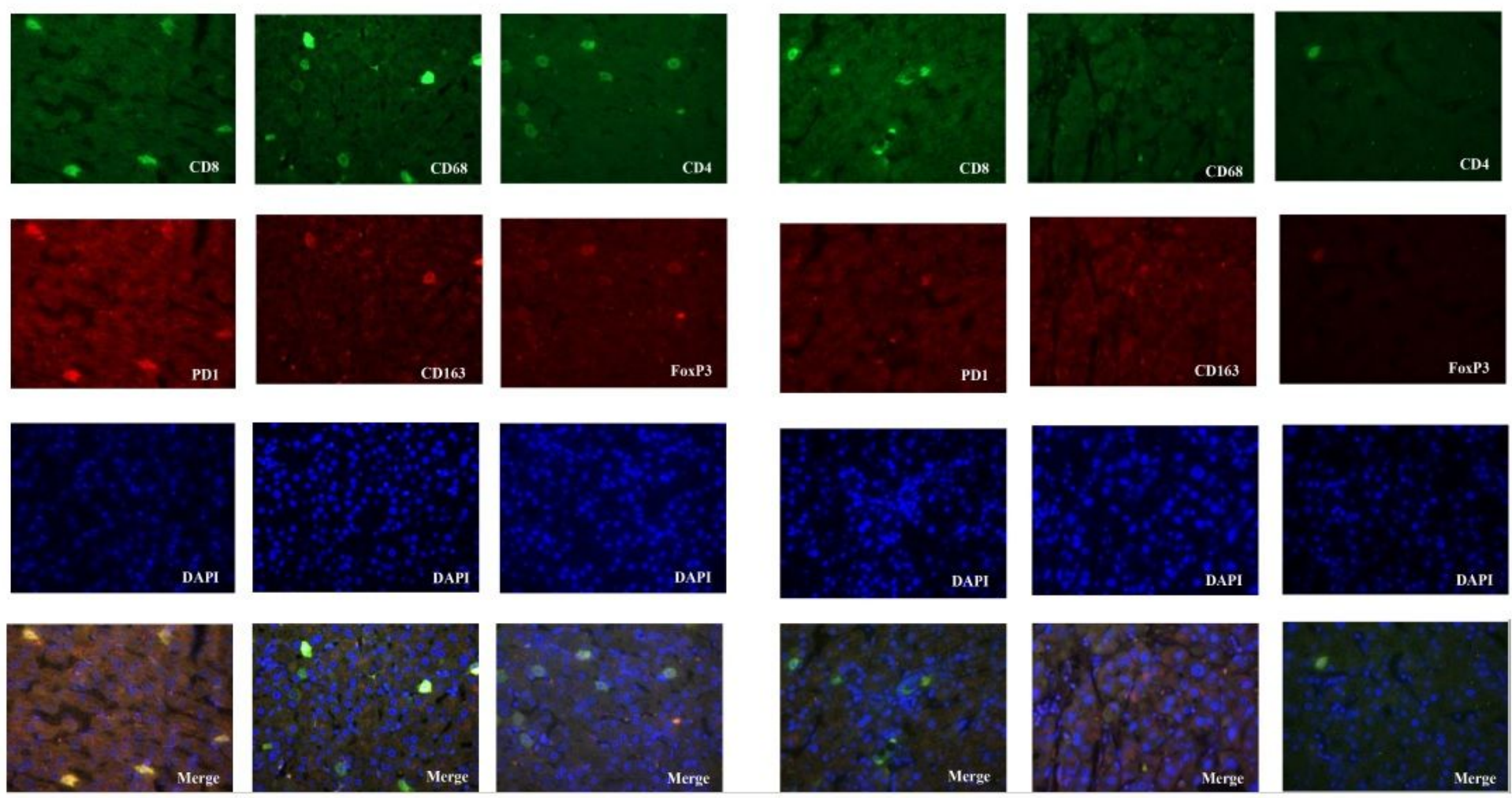

Figure 4

Immunofluorescence staining to analyze the TME of HCC.
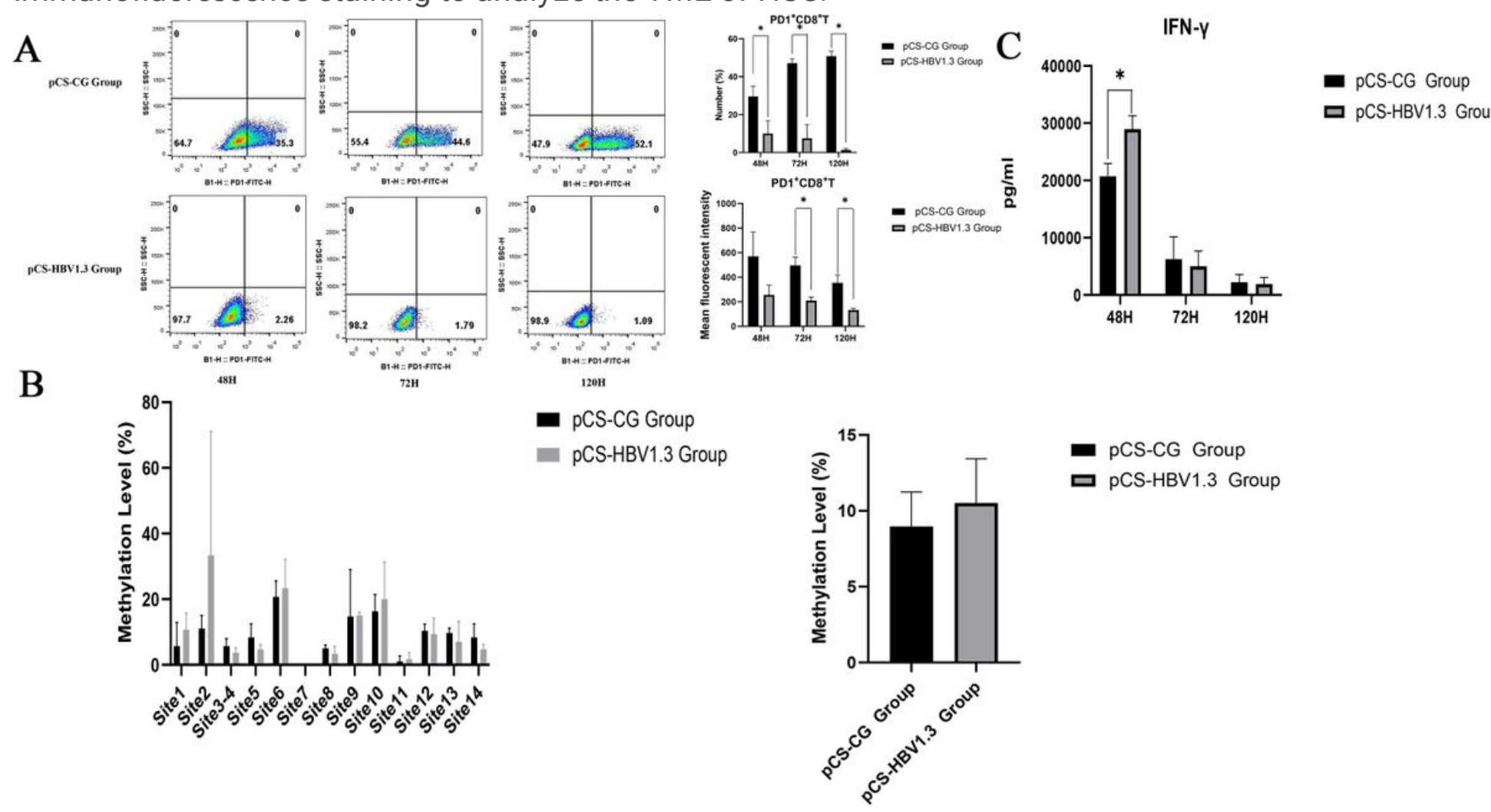
- pCS-CG Group
口 pCS-HBV1.3 Group

Figure 5 
The expressional change of PD-1 on cell membrane of CD8+ T cells upon invitro HBV infection. A.

Detection the expression of PD-1 on CD8+ T cells by Flow cytometric analysis in pCS-HBV1.3 group and pCS-CG group; B. Sequenom MassARRAY analysis of PD-1 gene methylation in pCS-HBV1.3 group and pCS-CG group; C. Cytokine measurement in PCS-HBV1.3 group and PCS-CG group.

\section{Supplementary Files}

This is a list of supplementary files associated with this preprint. Click to download.

- FigureS1.pdf

- Figures2.pdf

- TableS1.docx

- Tables2.docx 